\title{
Ferramenta computacional gráfico-iterativa de dimensionamento e detalhamento de vigas protendidas
}

Computational graphic-iterative tool of dimensioning and detailing of prestressed beams

\author{
A. C. S. Pacheco*; W. C. Lopes; M. W. Moura \\ Escola de Engenharia, Universidade Federal do Rio Grande - FURG, 96.203-000, Rio Grande-RS, Brasil \\ *aanapacheco@hotmail.com
}

\begin{abstract}
O presente trabalho traz um estudo que busca facilitar a solução de problemas de engenharia estrutural, com enfoque em vigas isostáticas de concreto protendido. Para tanto, foi desenvolvida uma ferramenta computacional em linguagem Visual Basic, ambiente de desenvolvimento de programas da Microsoft, que segue as disposições da NBR 6118 - Projeto de estruturas de concreto - procedimento. As rotinas do programa têm como foco realizar os cálculos envolvidos no dimensionamento e detalhamento de vigas protendidas. Em se tratando dos objetivos específicos, pode-se citar: cálculo das propriedades geométricas da seção transversal da viga; cálculo da força de protensão; dimensionamento da armadura de protensão; definição e traçado dos cabos de protensão, com exposição visual do desenho do cabo; cálculo das perdas de protensão; verificação das tensões em estado limite de serviço; verificação do estado limite último; verificação da flecha e; dimensionamento da armadura passiva. Os resultados são apresentados com a execução da ferramenta computacional, exposta aqui por figuras. A partir disso, concluiu-se que o dimensionamento de vigas protendidas pelo programa se mostra rápido e prático para solução de problemas, o que o faz útil para o trabalho na área de concreto protendido. A utilização das metodologias prescritas pelas normas vigentes permitiu análises detalhadas do comportamento em serviço das vigas.

Palavras-chave: concreto protendido, vigas, software.
\end{abstract}

The present work brings a study that intends to facilitate the solution of problems of structural engineering, focusing on isostatic beams of prestressed concrete. For this, a computational tool was developed in Visual Basic language, the Microsoft's program development environment, which follows the provisions of NBR 6118 - Concrete Structures Project - procedure. The program routines focus on performing the calculations involved in the sizing and detailing of prestressed beams. About the specific objectives, we can mention: calculation of the geometric properties of the beam's cross-section; calculation of the protension force; protension armature dimensioning; definition and tracing of the cables of pretension, with visual exhibition of the design of the cable; calculation of the loss of prestress; verification of the tensions in service limit state; verification of the ultimate limit state and; dimensioning of the passive reinforcement. The results are presented with the execution of the computational tool, presented here by figures. From this, it was concluded that the detailing of prestressed beams by the program is quick and practical for troubleshooting, which makes it useful for working in the prestressed concrete area. The use of the methodologies prescribed by the current norms allowed detailed analysis of the beam's in-service behavior.

Keywords: prestressed concrete, beams, software

\section{INTRODUÇÃO}

Os estudos acadêmicos que buscam soluções para a questão do cálculo estrutural por meio de ferramentas computacionais são recorrentes. Os programas desenvolvidos facilitam a solução de problemas, de forma que otimizam o tempo e diminuem a probabilidade de erros. Contudo, a maioria dos programas existentes são para estruturas de concreto armado, o que se explica pela maior facilidade de cálculo e execução desse sistema convencional. Esse projeto, em contrapartida, visa desenvolver uma ferramenta computacional para concreto protendido que servirá de base para projetos futuros.

De acordo com Pfeil (1988) [6], o emprego de armaduras protendidas no concreto é amplamente difundido nos dias de hoje. Essa técnica construtiva consiste na introdução de tensões prévias de compressão em uma estrutura nas regiões onde as solicitações de carregamento produzem tensões de tração. Isso proporciona um melhor desempenho da peça estrutural, visto que a resistência à tração é sempre desprezada nos cálculos. 
Sendo assim, objetivo principal do trabalho é desenvolver um programa computacional para dimensionar e detalhar vigas protendidas. Como objetivos específicos, pode-se citar o cálculo das propriedades geométricas da seção transversal da viga; cálculo da força de protensão; dimensionamento da armadura de protensão; definição e traçado dos cabos de protensão com exposição visual do desenho dos cabos; cálculo das perdas de protensão, verificação das tensões em estado limite de serviço, verificação das tensões em estado limite último, verificação da flecha e dimensionamento da armadura passiva.

Alguns trabalhos anteriores serviram de base, dentre os quais pode-se ressaltar Schwingel (1995) [7], Klein (2001) [10], Zanette (2006) [11], Momm (2004) [12], Korerich (2004) [13] e Moura (2004) [4].

\section{MATERIAL E MÉTODOS}

O programa foi desenvolvido em linguagem Visual Basic, através do Microsoft Visual Studio. As propriedades geométricas das vigas são calculadas pelo Teorema de Green, de modo a permitir o cálculo das propriedades geométricas de qualquer seção transversal. Já os cálculos empregados nas rotinas para o dimensionamento e verificação da estrutura seguem as disposições da NBR 6118 (2014) [5].

\subsection{CÁLCULO DAS PROPRIEDADES GEOMÉTRICAS}

Para o cálculo das propriedades geométricas da seção das vigas, optou-se pela utilização do Teorema de Green, que consiste em facilitar o método de integração transformando uma integral de superfície em uma integral de contorno. Dessa forma, basta o conhecimento prévio das coordenadas dos vértices para calcular as propriedades geométricas de qualquer tipo de seção, considerando uma poligonal cujas coordenadas dos vértices são dispostas em sentido anti-horário em relação a um sistema de eixos conforme a Figura 1.

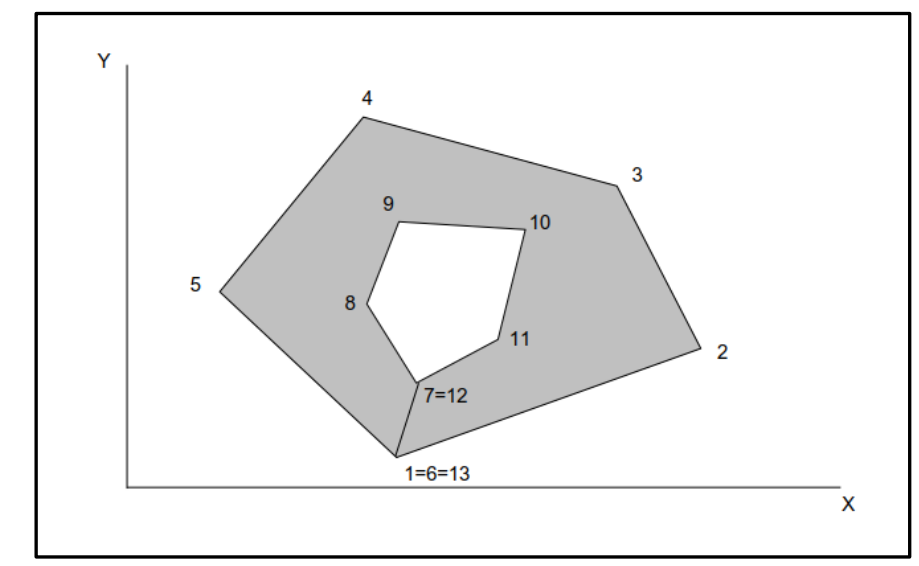

Figura 1: Disposição das coordenadas no Teorema de Green [2].

\subsection{CÁlCULO DA FORÇA DE PROTENSÃo}

Para o cálculo da força de protensão final, é feito através uma estimativa do fator de perdas de protensão arbitrado pelo projetista desde que se garanta a verificação de determinados estados limites de serviço. A partir da Equação 1 estima-se o valor da força inicial de protensão.

$$
P i_{e s t}=\frac{P_{\infty}}{r_{\infty}}
$$

Onde:

$P i_{\text {est }}=$ força de protensão estimada.

$P_{\infty}=$ força de protensão da verificação dos estados limites de serviço. 
$r_{\infty}=$ fator de perdas de protensão estimadas.

A partir da força de protensão estimada, é calculada a área de protensão estimada a partir da Equação 2, no qual $\sigma_{p i}$ é a máxima tensão que atua no aço.

$$
A p_{e s t}=\frac{P i_{e s t}}{\sigma_{p i}}
$$

O dimensionamento da armadura de protensão, ou seja, a definição do número mínimo de cordoalhas, $N_{\min }$, é dado em função da área de uma unidade da armadura, por exemplo, uma barra ou uma cordoalha, esta área unitária recebe o nome, $A_{p 1}$, e, conforme a Equação 3.

$$
N_{\min }=\frac{A p_{\text {est }}}{A_{p 1}}
$$

Definido o número mínimo de cordoalhas, é indicado o número de cabos, $N_{\text {cabos }}$, e de cordoalhas por cabo, $N_{\text {cord } / c a b o}$. Assim, calcula-se a área de aço protendido existente, e, em seguida, a força de protensão inicial utilizada, conforme as Equações 4 e 5 .

$$
\begin{gathered}
\text { Ap, exist }=N_{\text {cabos }} \times N_{\text {cord } / \text { cabo }} \times A_{p 1} \\
P_{i}=\sigma_{p i} \times A p, \text { exist }
\end{gathered}
$$

\subsection{TRAÇADO DOS CABOS}

A NBR 6118 (2014) [5] diz que a armadura de protensão pode ser retilínea, curvilínea, poligonal ou de traçado misto. Deve ser projetada de modo que atue em sentido oposto aos esforços devido ao carregamento externo produzido e visando também limitar as perdas de protensão.

No programa computacional é permitido que sejam utilizados cabos retos e parabólicos. O traçado dos cabos é mostrado a partir de três abcissas e ordenadas informadas pelo usuário: as de extremo e meio do vão. É mostrado, também, o traçado do cabo equivalente, calculado a partir da média das ordenadas dos cabos nas diversas seções da viga.

\subsection{PERDAS DE PROTENSÃO}

As perdas de protensão se referem à diminuição que ocorre no valor da força de protensão inicialmente aplicada em uma estrutura. São divididas em perdas imediatas - que ocorrem no momento do ato da protensão -, e perdas progressivas - que ocorrem ao longo do tempo.

O cálculo realizado no programa computacional considerou tração pelas duas extremidades da viga e utilizou o método do cabo equivalente, e analisa as perdas de protensão ao longo de 11 seções transversais. O método do cabo equivalente, segundo Moura (2004) [4], pode representar de maneira satisfatória o comportamento da viga no centro do vão, onde os cabos estão próximos.

\subsubsection{PERDA POR ATRITO}

A primeira perda de protensão ocorre devido à força de atrito gerada pelos pontos de contato dos cabos de protensão com a bainha metálica em que estão inseridos. Dessa forma, parte da força de tração aplicada no cabo pelo macaco é consumida para vencer essa solicitação.

Conforme Collins e Mitchell (1987) [14], o contato dos cabos com a bainha se dá principalmente em trechos de curvatura do traçado dos cabos, sendo a perda nessa situação chamada de perda por atrito em curva. Porém, também ocorre perda em trechos retilíneos devido à sinuosidade das bainhas, sendo neste caso denominada perda por ondulação parasita. A perda total de atrito é calculada pela soma da perda por atrito em curva com a perda por ondulação parasita, conforme a Equação 6. 


$$
P_{a t}(x)=P_{i} \times\left[e^{-\mu\left(\sum \alpha+0,01 x\right)}\right]
$$

Onde:

$P_{a t}(x)=$ força de protensão o ao longo do cabo após a perda por atrito.

$P_{i}=$ força inicial de protensão.

$\mu=$ coeficiente de atrito aparente entre o cabo e a bainha.

$\alpha$ = ângulo entre a posição "x" e a origem.

\subsubsection{PERDA POR ACOMODAÇÃO NAS ANCORAGENS}

A perda por acomodação nas ancoragens ocorre devido a uma diminuição de alongamento do cabo no momento de transferência da força de protensão do macaco para a ancoragem. Este retorno do cabo, que causa perda de alongamento, está apresentado na Figura 2.

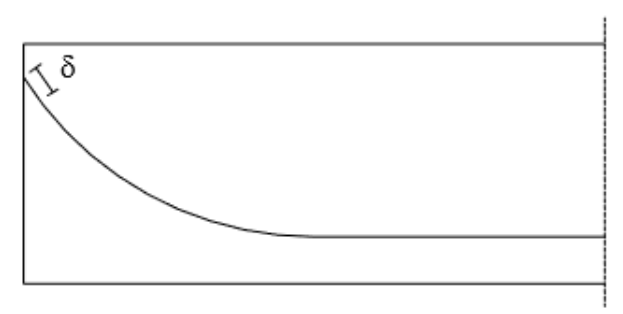

Figura 2: Perda por acomodação nas ancoragens.

\subsubsection{PERDA POR ENCURTAMENTO ELÁSTICO DO CONCRETO}

A perda por encurtamento elástico ocorre devido a aplicação da força de compressão na peça que gera um encurtamento da mesma. Conforme os cabos são tracionados, um de cada vez, a deformação do concreto provocada pela força no cabo que está sendo tracionado gera perda de tensão nos cabos já ancorados. Pode-se dizer, assim, que a alteração da deformação da armadura de protensão é equivalente à deformação do concreto. $\mathrm{O}$ cálculo das perdas por encurtamento elástico do concreto é feito pela Equação 7.

$$
\Delta \sigma_{e n c}=\alpha_{p} \times\left(\sigma_{c p}(x)+\sigma_{c g}(x)\right) \times \frac{n-1}{2 n}
$$

Onde:

$\Delta \sigma_{e n c}=$ variação de tensão causada pelo encurtamento elástico.

$n=$ número de cabos.

$\alpha_{p}=$ relação entre os módulos de elasticidade do aço e do concreto.

$\sigma_{c p}(x)=$ tensão inicial no concreto ao longo do cabo devida à protensão simultânea dos cabos.

$\sigma_{c g}(x)=$ tensão no concreto ao longo do cabo devida à carga permanente mobilizada pela protensão.

\subsubsection{PERDAS PROGRESSIVAS}

As perdas progressivas, também chamadas de perdas diferidas, ocorrem ao longo da vida útil da estrutura. No concreto ocorre a retração e fluência, que correspondem respectivamente à diminuição do volume da peça devido à perda de parte da água de amassamento e ao aumento gradual de deformação devido à ação de carga constante. Já no aço ocorre a relaxação, efeito este correspondente à diminuição da tensão no material, que ocorre quando a armadura, deformada por uma solicitação inicial, é mantida com comprimento constante.

A metodologia utilizada no programa foi o processo aproximado apresentado na NBR 6118 (2014) [5], conforme a Equação 8. 


$$
P_{\text {pro }}(x)=P_{e n c}(x) \times\left\{1-0,01 \times\left[7,4+\alpha_{p} \times \frac{\phi^{1,07}}{18,7} \times\left(3+\sigma_{c, p o g}(x)\right)\right]\right\}
$$

Onde:

$P_{\text {pro }}(x)=$ força de protensão ao longo do cabo após as perdas progressivas.

$P_{e n c}(x)=$ força de protensão ao longo do cabo após a perda por encurtamento elástico do concreto. $\phi=$ coeficiente de fluência do concreto.

$\sigma_{c, p o g}(x)=$ tensão no concreto adjacente ao cabo resultante, provocada pela protensão e carga permanente mobilizada.

\subsection{ESTADOS LIMITES DE SERVIÇO}

Ao contrário do que ocorre em concreto armado, em concreto protendido o dimensionamento da armadura protendida dos elementos é feito pelos estados limites de serviço e a partir disso são realizadas as verificações para o estado limite último. As vigas protendidas devem respeitar os estados limites de descompressão, de compressão excessiva e de formação de fissuras entre outros para boa funcionalidade. Para o cálculo dessas verificações, é necessário que se saiba o grau de protensão, as tensões admissíveis e que sejam definidas as combinações de ações pelo projetista.

No programa computacional, foram contemplados até o momento, os casos de protensão completa e limitada. As verificações são feitas conforme os itens 2.5.1 e 2.5.2.

\subsubsection{PROTENSÃO COMPLETA}

No caso de protensão completa, devem ser atendidas as seguintes condições:

a) para as combinações frequentes de ações, previstas no projeto, é respeitado o estado limite de descompressão, conforme a Equação 9.

$$
\sigma_{c 1}=P_{\infty} \times\left(\frac{1}{A}+\frac{e_{p}}{W_{i n f}}\right)-\frac{M_{g}+\Psi_{1} \times M_{q}}{W_{i n f}} \leq 0
$$

Onde:

$\sigma_{c 1}=$ tensão normal no bordo inferior.

$A=$ área da seção transversal do concreto.

$e_{p}=$ excentricidade de protensão.

$W_{\text {inf }}=$ módulo resistente inferior.

$M_{g}=$ momento devido à carga permanente.

$M_{q}=$ momento devido à carga acidental.

$\Psi_{1}=$ coeficiente de redução para combinações frequentes de ações.

b) para as combinações raras de ações, previstas no projeto, é respeitado o estado limite de formação de fissuras, conforme a Equação 10.

$$
\sigma_{c 1}=P_{\infty} \times\left(\frac{1}{A}+\frac{e_{p}}{W_{i n f}}\right)-\frac{M_{g}+M_{q}}{W_{i n f}} \leq f_{c t, f}
$$

Sendo $f_{c t, f}$ a resistência à tração na flexão.

c) para as combinações raras de ações, previstas no projeto, é respeitado o estado limite de compressão excessiva na borda superior, conforme a Equação 11.

$$
\sigma_{t 1}=P_{\infty} \times\left(\frac{1}{A}+\frac{e_{p}}{W_{\text {sup }}}\right)-\frac{M_{g}+M_{q}}{W_{\text {sup }}} \geq-0,7 \times f_{c k}
$$


d) para as combinações raras de ações, previstas no projeto, é respeitado também o estado limite de compressão excessiva na borda inferior, conforme a Equação 12.

$$
\sigma_{t 1}=P_{\infty} \times\left(\frac{1}{A}+\frac{e_{p}}{W_{i n f}}\right)-\frac{M_{g}+M_{q}}{W_{i n f}} \geq-0,7 \times f_{c k}
$$

\subsubsection{PROTENSÃO LIMITADA}

No caso de protensão limitada, devem ser atendidas as seguintes condições:

a) para as combinações quase-permanentes de ações, previstas no projeto, é respeitado o estado limite de descompressão, conforme a Equação 13;

$$
\sigma_{c 1}=P_{\infty} \times\left(\frac{1}{A}+\frac{e_{p}}{W_{i n f}}\right)-\frac{M_{g}+\Psi_{2} \times M_{q}}{W_{i n f}} \leq 0
$$

Sendo $\Psi_{2}$ o coeficiente de redução para combinações quase-permanentes de ações.

b) para as combinações frequentes de ações, previstas no projeto, é respeitado o estado limite de formação de fissuras, conforme a Equação 14.

$$
\sigma_{c 1}=P_{\infty} \times\left(\frac{1}{A}+\frac{e_{p}}{W_{i n f}}\right)-\frac{M_{g}+\Psi_{1} \times M_{q}}{W_{i n f}} \leq f_{c t, f}
$$

c) para as combinações frequentes de ações, previstas no projeto, é respeitado o estado limite de compressão excessiva na borda superior, conforme a Equação 15.

$$
\sigma_{t 1}=P_{\infty} \times\left(\frac{1}{A}+\frac{e_{p}}{W_{\text {sup }}}\right)-\frac{M_{g}+\Psi_{1} \times M_{q}}{W_{\text {sup }}} \geq-0,7 \times f_{c k}
$$

d) para as combinações quase permanentes de ações, previstas no projeto, é respeitado também o estado limite de compressão excessiva na borda superior, conforme a Equação 16.

$$
\sigma_{t 1}=P_{\infty} \times\left(\frac{1}{A}+\frac{e_{p}}{W_{\text {sup }}}\right)-\frac{M_{g}+\Psi_{2} \times M_{q}}{W_{\text {sup }}} \geq-0,7 \times f_{c k}
$$

\subsection{ESTADO LIMITE ÚLTIMO}

O estado limite último é o estado que, pela sua simples ocorrência, determina a paralisação, no todo ou em parte, do uso da construção. Para a sua verificação, primeiramente calcula-se as tensões máximas que podem ser atingidas de compressão $\left(\sigma_{c}{ }^{\max }\right)$ e de tração $\left(\sigma_{t}{ }^{\max }\right)$, conforme as Equações 17 e 18.

$$
\begin{gathered}
\sigma_{c}^{\max }=\left|0,7 \times f_{c k j}\right| \\
\sigma_{t}^{\text {max }}=\left|1,2 \times f_{c t m j}\right|
\end{gathered}
$$

Onde:

$f_{c k j}=$ resistência do concreto à compressão aos j dias de idade.

$f_{c t m j}=$ resistência média do concreto à tração.

Com isso, é utilizada a combinação normal para o cálculo das tensões normais nas bordas superior e inferior ao longo da viga, conforme as Equações 19 e 20. 


$$
\begin{aligned}
& \sigma_{2}=\gamma_{p} \times P_{\text {enc }}(x)\left(\frac{1}{A}+\frac{e_{p}}{W_{\text {sup }}}\right)-\frac{M_{g 1}}{W_{\text {sup }}} \\
& \sigma_{1}=\gamma_{p} \times P_{\text {enc }}(x)\left(\frac{1}{A}+\frac{e_{p}}{W_{\text {inf }}}\right)-\frac{M_{g 1}}{W_{\text {inf }}}
\end{aligned}
$$

Onde:

$\sigma_{2}=$ tensão normal na borda superior.

$\sigma_{1}=$ tensão normal na borda inferior.

$\gamma_{p}=$ coeficiente de ponderação para combinação normal.

Uma vez que todas as tensões estão dentro dos limites máximos, a estrutura confere.

\subsection{VERIFICAÇÃO DA FLECHA}

O cálculo da flecha inicial da viga $(\delta)$ é mostrado na Equação 22, e é feito considerando o momento $(q)$ como a seguinte combinação:

$$
\begin{gathered}
q=\left(g_{1}+g_{2}\right)+\varphi \times q \\
\delta=\frac{5}{384} \frac{q \times L^{4}}{E I}
\end{gathered}
$$

Onde:

$g_{1}=$ carga devido ao peso próprio.

$g_{2}=$ carga permanente.

$E=$ módulo de elasticidade.

$I$ = momento de inércia da seção da viga.

A flecha que ocorrerá após as considerações ao longo do tempo $\left(\delta_{\infty}\right)$ se dará como segue.

$$
\begin{gathered}
P_{p}=\frac{8 \times P_{\text {pro }}(x) \times e_{p}}{L^{2}} \\
\delta_{p}=\frac{5}{384} \frac{P_{p} \times L^{4}}{E I} \\
\delta_{0}=\delta-\delta_{p} \\
\delta_{\infty}=\delta_{0}(1+\phi)
\end{gathered}
$$

Onde:

$P_{p}=$ carregamento equivalente que solicita as seções da viga do mesmo modo que o cabo protendido.

$\delta_{p}=$ flecha devido ao carregamento equivalente ao cabo protendido.

Por fim, deve-se comparar a flecha obtida com a flecha admissível $\left(\delta_{a d m}\right)$.

$$
\delta_{a d m}=\frac{L}{250}
$$

\subsection{DIMENSIONAMENTO DA ARMADURA PASSIVA}

Como já foi mencionado anteriormente, a armadura protendida da viga é calculada de forma a atender as exigências dos estados limites de serviço. Portanto, calcula-se também uma armadura passiva de forma a assegurar o equilíbrio na ruptura. 


\subsubsection{ARMADURA LONGITUDINAL}

Para o dimensionamento da armadura longitudinal, primeiramente se faz um equilíbrio de momentos na seção para encontrar a posição da linha neutra $(x)$, conforme simplificado na Equação 28.

$$
-0,32 \sigma_{c d} b_{f} x^{2}+0,8 \sigma_{c d} b_{f} d-A_{p} f_{p y d}\left(d p-d^{\prime}\right)-M_{d}=0
$$

Onde:

$b_{f}=$ largura da mesa.

$\sigma_{c d}=$ tensão de compressão do concreto.

$d^{\prime}=$ cobrimento da armadura.

$d p=h-e_{p}$

$d=$ altura útil da seção.

$f_{\text {pyd }}=$ resistência de escoamento do aço da armadura ativa.

$M_{d}=$ momento de projeto devido às cargas permanente e acidental.

Com isso, é possível encontrar a área de armadura longitudinal $\left(A_{s 1}\right)$ e fazer uma comparação dela com a armadura mínima necessária $\left(A_{s m i n}\right)$, conforme as Equações 29 e 30.

$$
\begin{gathered}
A_{s 1}=\frac{0,8 \times x \times b_{f} \times \sigma_{c d}-A_{p} f_{p y d}}{f_{y d}} \\
A_{\text {smin }}=0,8 \times \xi \times b_{f} \times d \times \frac{\sigma_{c d}}{f_{y d}}
\end{gathered}
$$

Sendo $f_{y d}$ a resistência de cálculo do aço.

\subsubsection{ARMADURA TRANSVERSAL}

Primeiramente, verifica-se as bielas de compressão com a condição de que a tensão cisalhante de cálculo $\left(\tau_{w d}\right)$ deve ser menor que a tensão cisalhante limite $\left(\tau_{w u}\right)$, sendo que essas tensões são calculadas conforme as Equações 31 e 32 .

$$
\begin{aligned}
\tau_{w d} & =\frac{1,4 \times\left(V_{g}+V_{q}\right)-0,9 V_{p}}{b_{w} \times f_{y p d}} \\
\tau_{w u} & =0,27 \times\left(1-\frac{f_{c k}}{250}\right) \times f_{c d}
\end{aligned}
$$

Onde:

$b_{w}=$ largura da alma.

$V_{g}=$ esforço cortante devido ao carregamento permanente.

$V_{q}=$ esforço cortante devido ao carregamento acidental.

$V_{p}=$ esforço cortante gerado pela curvatura do cabo de protensão.

Por fim, encontra-se a área de armadura transversal $\left(A_{s w}\right)$ e se compara ela com a armadura mínima necessária $\left(A_{\text {smin }}\right)$, conforme as Equações 33 e 34 .

$$
\begin{aligned}
A_{s w} & =100 b_{w} \tau_{d} f_{y d} \\
A_{s w \text { min }} & =\frac{\rho_{\text {min }} \times 100 \times b_{w}}{100}
\end{aligned}
$$


Sendo $\tau_{d}$ a tensão convencional de cisalhamento.

\section{VERIFICAÇÃO DO MODELO PROPOSTO}

Com o objetivo de validar os resultados obtidos pelo programa, foi realizado um cálculo manual utilizando as equações apresentadas anteriormente. Os dados utilizados para os cálculos são exibidos abaixo:

Tipo de seção: T ou outra;

Comprimento da viga: $30 \mathrm{~m}$;

Carregamento permanente principal: $26,57 \mathrm{kN} / \mathrm{m}$;

Carregamento permanente secundário: $22 \mathrm{kN} / \mathrm{m}$;

Carregamento acidental: $22,5 \mathrm{kN} / \mathrm{m}$;

Tipo de protensão: limitada;

Fck: $40 \mathrm{MPa}$;

Data de protensão: 15 dias;

Tipo de aço de protensão: CP 190 RB 15,2 mm;

Área de uma cordoalha: $1,4 \mathrm{~cm}^{2}$;

Fator de perdas estimadas: 0,75 (25\%);

Atrito entre a barra e bainha metálica: 0,2 .

Inicialmente, a força final de protensão foi calculada através das Equações 12 e 13 . O maior valor encontrado foi: $P_{\infty}=-5858,10 \mathrm{kN}$, chegando a uma força de protensão estimada igual a $P i_{e s t}=-5858,10 / 0,75=-7810,80 \mathrm{kN}$. A partir disto, estimou-se a área de aço necessária, considerando uma tensão $\sigma_{p i}=140,2 \mathrm{kN} / \mathrm{cm}^{2}$, resultando em $A p_{e s t}=55,70 \mathrm{~cm}^{2}$. Sendo a área da cordoalha $A_{p 1}=1,4 \mathrm{~cm}^{2}$, calculou-se através da Equação 3 o número mínimo de cordoalhas necessárias, sendo este $N_{\text {mín }}=40$ (arredondando para cima). Deste modo, adotando quatro cabos com dez cordoalhas cada, a área existente do aço é $A p=56 \mathrm{~cm}^{2}$, conforme Equação 4. Finalmente, a força de protensão foi calculada pela Equação 5, resultando em $P_{i}=-7852,32 \mathrm{kN}$.

De posse do valor de $P_{i}$, foram calculadas as perdas de protensão. De modo a ilustrar os cálculos feitos, tomou-se a seção $0-\operatorname{com} x=0 m$ - como exemplo. Em razão disso, calculou-se inicialmente as perdas por atrito, através da Equação 10, como é apresentado:

$$
P_{a t}(x=0)=-7852,32 \times\left[e^{-0,2(0+0,01 \times 0)}\right]=-7852,32 k N
$$

Feito isso, calculou-se a perda por acomodação das ancoragens, considerando que o ponto de repouso do cabo é menor que a metade do vão. Logo:

$$
P_{a c}(x=0)=-(-7852,32)-2 \times(7852,32-7475,99)=-7099,66 k N
$$

A próxima perda calculada foi a provocada pelo encurtamento elástico da peça de concreto $P_{e n c}(x)$. O cálculo foi feito segundo a Equação 7 , levando em conta que $\alpha_{p}=E_{p} / E_{c s}=$ $19500 / 3187,58=6,12$ e que a soma das tensões $\sigma_{c p}$ e $\sigma_{c g}$ é dada por:

$$
\begin{gathered}
\sigma_{c p}(x)+\sigma_{c g}(x)=P_{a c}(x) \times\left(\frac{1}{A}+\frac{y(x)^{2}}{I_{g x}}\right)-\frac{M_{g 1} \times y(x)}{I_{g x}} \\
\sigma_{c p}(x=0)+\sigma_{c g}(x=0)=-7099,66 \times\left(\frac{1}{10626,93}\right)=-0,67 \mathrm{kN} / \mathrm{cm}^{2} \\
P_{\text {enc }}(x=0)=-7099,66-\left[56 \times 6,12 \times(-0,67) \times \frac{4-1}{2 \times 4}\right]=7013,55 \mathrm{kN}
\end{gathered}
$$


Por último, as perdas progressivas foram calculadas de acordo com a Equação 8, sendo $\sigma_{c, p o g}(x)$ dada por:

$$
\begin{gathered}
\sigma_{c, p o g}(x)=P_{e n c}(x) \times\left(\frac{1}{A}+\frac{y(x)^{2}}{I_{g x}}\right)-\frac{\left(M_{g 1}+M_{g 2}\right) \times y(x)}{I_{g x}} \\
\sigma_{c, p o g}(x=0)=-7013,55 \times\left(\frac{1}{10626,93}\right)=-0,66 \mathrm{kN} / \mathrm{cm}^{2}
\end{gathered}
$$

Deste modo, adotando $\phi=2,5$, determina-se a força de protensão após todas as perdas.

$$
P_{\text {pro }}(x=0)=-7013,55 \times\left\{1-7,4+6,12 \times \frac{2,5^{1,07}}{18,7} \times[3-(-6,6)] / 100\right\}=-5907,17 \mathrm{kN}
$$
eles:

Após os cálculos das perdas, foram feitas as verificações dos estados limites de serviço, sendo

Verificação 1: Descompressão para a combinação quase permanente;

Verificação 2: Formação de fissuras para a combinação frequente;

Verificação 3: Compressão excessiva para a combinação frequente;

Verificação 4: Compressão excessiva para combinação quase permanente.

Foram feitas as verificações para a seção 5, a fim de exemplificar o problema.

Verificação 1:

$$
\begin{gathered}
-6176,72 \times\left(\frac{1}{10626,93}+\frac{-71,89}{-410873,02}\right)-\frac{(546412,5+0,4 \times 253125)}{-410873,02} \leq 0 \\
-0,085 \frac{k N}{c m^{2}} \leq 0 \rightarrow O K !
\end{gathered}
$$

Verificação 2:

$$
\begin{gathered}
-6176,72 \times\left(\frac{1}{10626,93}+\frac{-71,89}{-410873,02}\right)-\frac{(546412,5+0,6 \times 253125)}{-410873,02} \leq 2,95 \\
0,037 \mathrm{kN} / \mathrm{cm}^{2} \leq 2,95 \rightarrow O K !
\end{gathered}
$$

Verificação 3:

$$
\begin{gathered}
-6176,72 \times\left(\frac{1}{10626,93}+\frac{-71,89}{454299,79}\right)-\frac{(546412,5+0,6 \times 253125)}{454299,79} \leq-2,8 \\
-1,14 \mathrm{kN} / \mathrm{cm}^{2} \leq-2,8 \mathrm{kN} / \mathrm{cm}^{2} \rightarrow O K
\end{gathered}
$$

Verificação 4:

$$
\begin{gathered}
-6176,72 \times\left(\frac{1}{10626,93}+\frac{-71,89}{454299,79}\right)-\frac{(546412,5+0,4 \times 253125)}{454299,79} \leq-2,8 \\
-1,029 \mathrm{kN} / \mathrm{cm}^{2} \leq-2,8 \mathrm{kN} / \mathrm{cm}^{2} \rightarrow O K
\end{gathered}
$$

Para a verificação do estado limite último, primeiro foram calculadas as tensões máximas de tração e compressão:

$$
\sigma_{c}^{\max }=|0,7 \times 0,8 \times 40|=22,4 M P a
$$




$$
\sigma_{t}^{\max }=\left|1,2 \times 0,8 \times 0,3 \times 40^{2 / 3}\right|=3,3685 \mathrm{MPa}
$$

Tomou-se a seção 0 como exemplo, sendo encontrados os seguintes valores de tensões normais, ambos dentro dos limites:

$$
\begin{aligned}
& \sigma_{2}=10 \times 1,1 \times-7013,55\left(\frac{1}{10626,93}\right)=-7,26 M P a \\
& \sigma_{1}=10 \times 1,1 \times-7013,55\left(\frac{1}{10626,93}\right)=-7,26 M P a
\end{aligned}
$$

Quanto à flecha, foi feito o seu cálculo e comparado com a flecha admissível, dada por $\delta_{a d m}=$ $3000 / 250=12 \mathrm{~cm}$.

$$
\begin{gathered}
q=(26,57+22)+0,4 \times 22,5=57,57 \\
\delta=\frac{5}{384} \frac{57,57 \times 30^{4}}{E I}=1,49 \\
P_{p}=\frac{8 \times-5907,65 \times-71,89}{30^{2}}=37,75 \\
\delta_{p}=\frac{5}{384} \frac{37,75 \times 30^{4}}{E I}=0,98 \mathrm{~cm} \\
\delta_{0}=1,49-0,98=0,51 \mathrm{~cm} \\
\delta_{\infty}=0,51(1+2,5)=1,78 \mathrm{~cm}
\end{gathered}
$$

\section{RESULTADOS E DISCUSSÃO}

Os resultados obtidos com o programa computacional são apresentados a partir dos dados de saída dele. A Figura 3 mostra a tela de entrada do programa desenvolvido e nesta já é possível calcular as propriedades geométricas da seção.

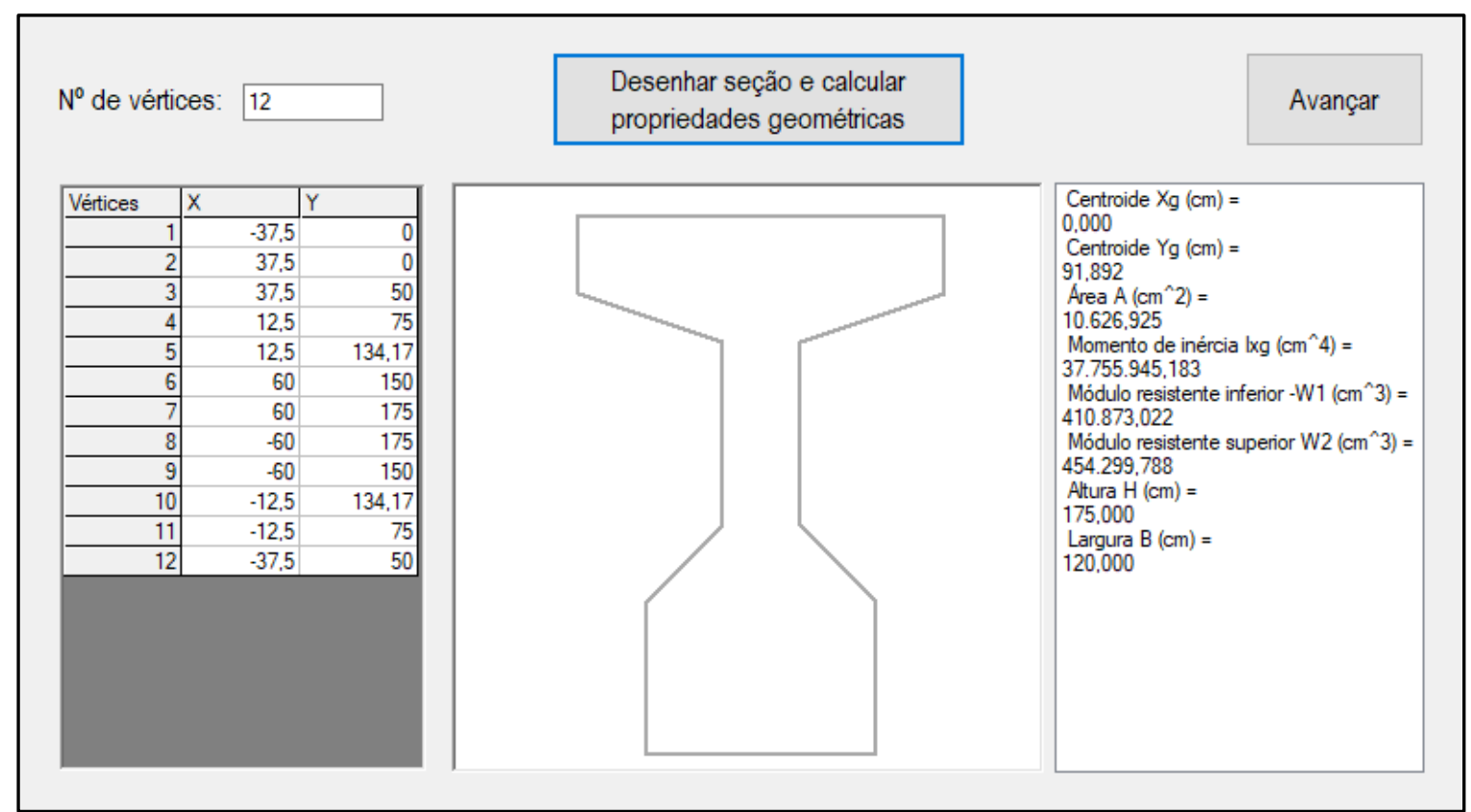

Figura 3: Cálculo das propriedades geométricas. 
A Figura 4 traz a tela onde se realiza o cálculo da força de protensão e, consequentemente, o dimensionamento da armadura de protensão com o respectivo número de cabos e de cordoalhas por cabo.

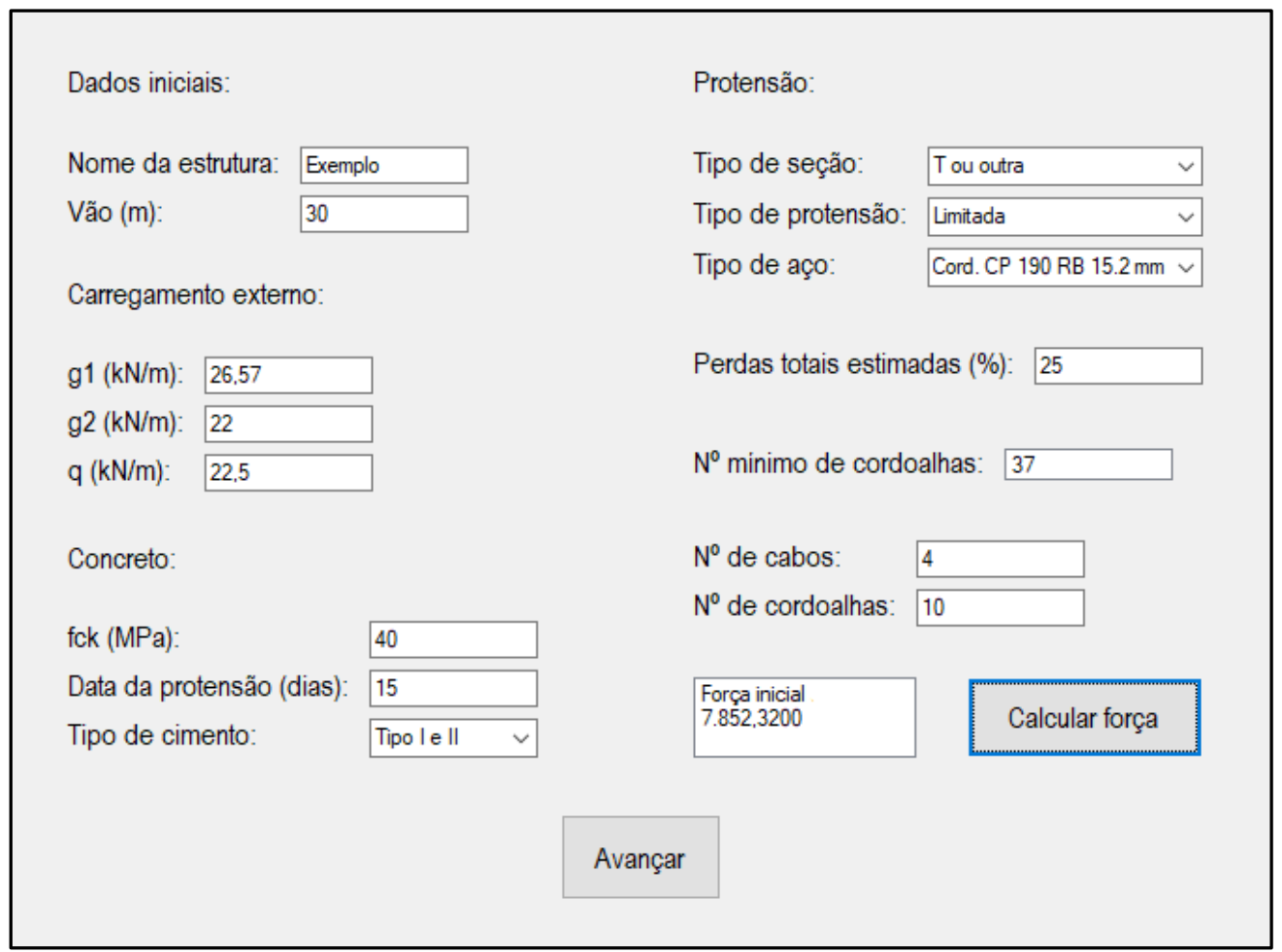

Figura 4: Cálculo da força de protensão.

A Figura 5 mostra a tela de entrada do traçado dos cabos de protensão. A partir daí o programa calcula o cabo equivalente e, então, são calculadas as perdas de protensão como mostra a Figura 6.

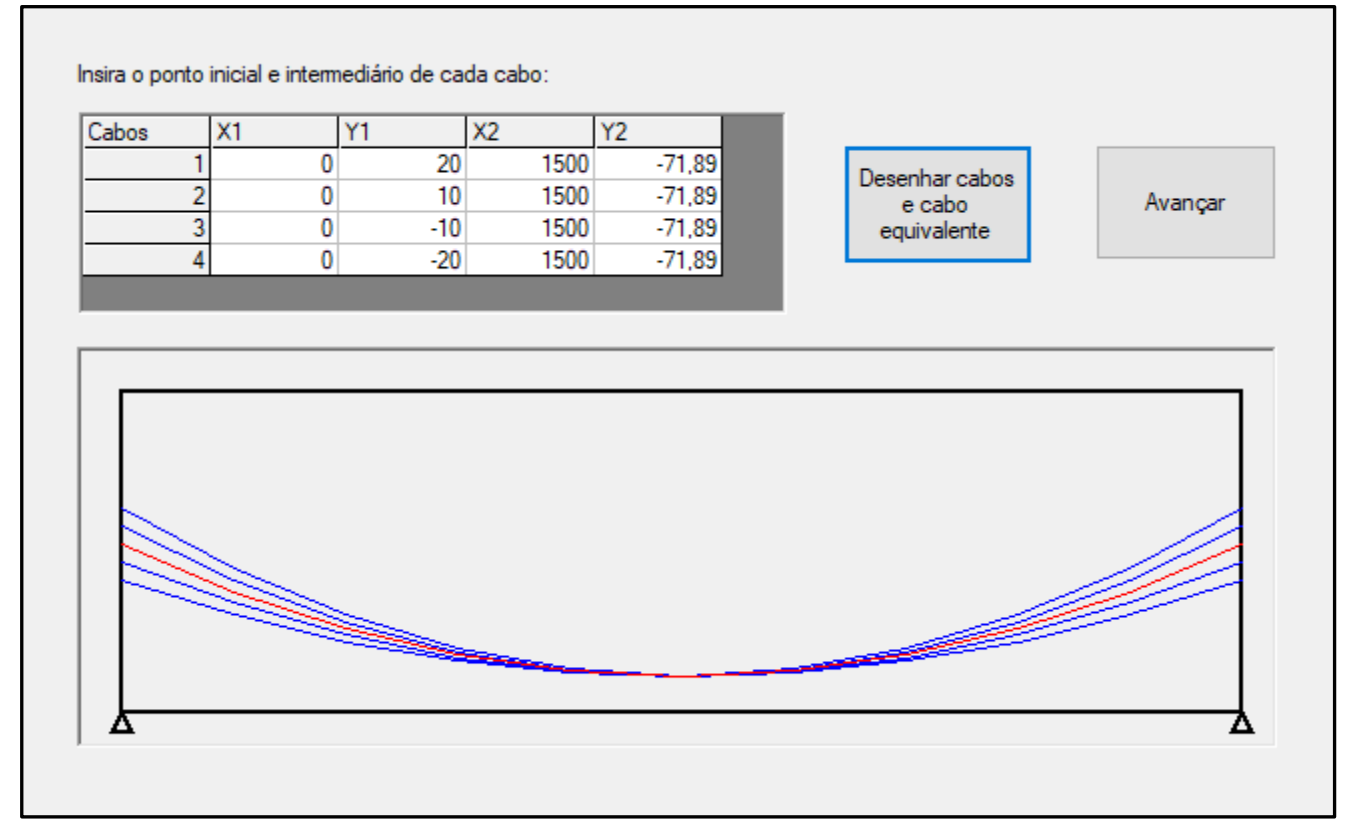

Figura 5: Traçado dos cabos. 


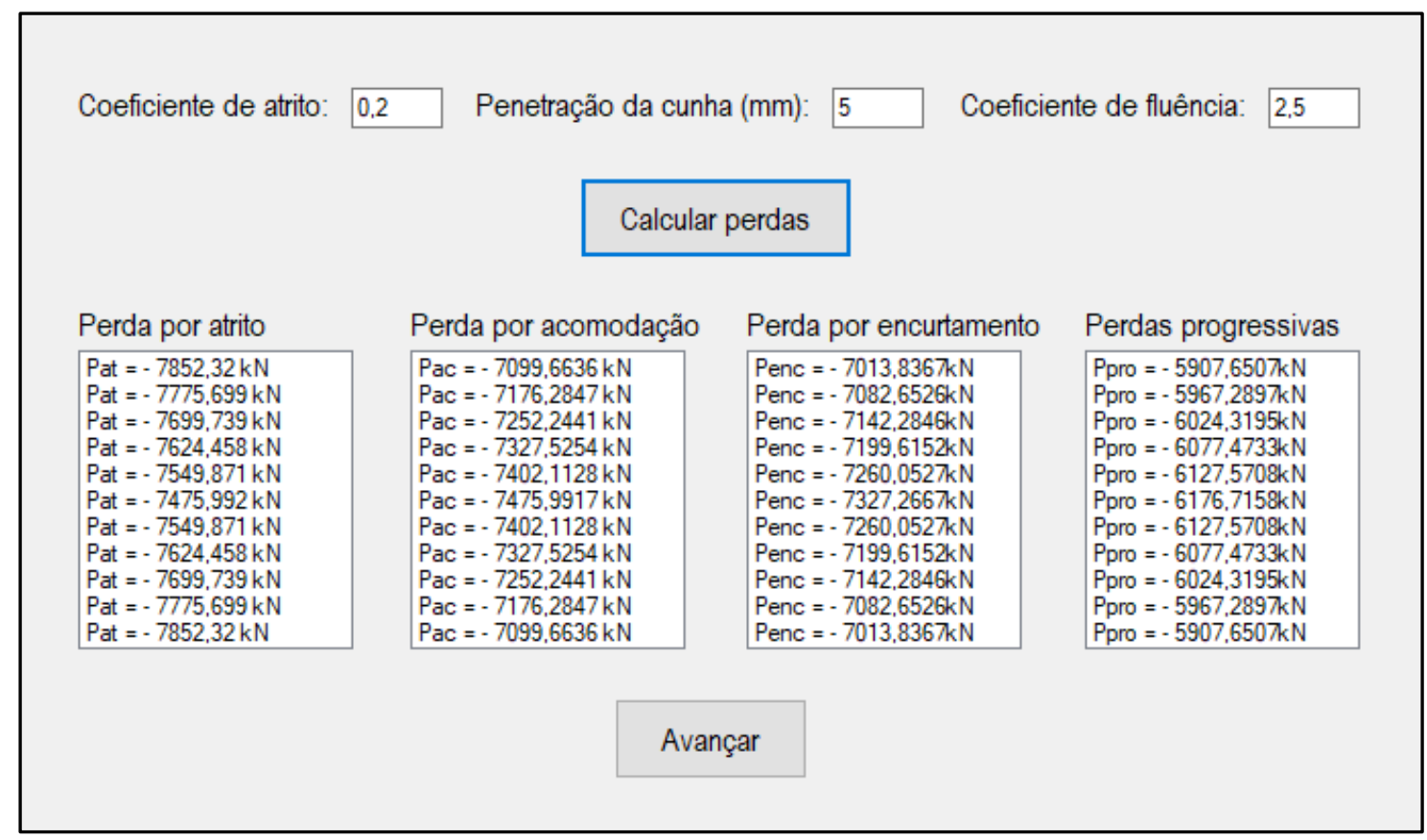

Figura 6: Cálculo das perdas de protensão.

A Figura 7 mostra os valores das tensões normais nos estados limites de serviço e verifica se estão dentro dos limites prescritos pela NBR 6118:2014 [5].

\begin{tabular}{|c|c|c|c|}
\hline \multicolumn{4}{|c|}{ Estado limite de serviço } \\
\hline Descompressão & Compressão excessiva & Formação de fissuras & Compressão excessiva \\
\hline \begin{tabular}{|l|} 
Combinação quase pemanente \\
$-0.5559 \mathrm{Ok}$ \\
$-0.3699 \mathrm{Ok}$ \\
$-0.2327 \mathrm{Ok}$ \\
$-0.141 \mathrm{Ok}$ \\
$-0.0926 \mathrm{Ok}$ \\
$-0.0857 \mathrm{Ok}$ \\
$-0.0926 \mathrm{Ok}$ \\
$-0.141 \mathrm{Ok}$ \\
$-0.2327 \mathrm{Ok}$ \\
$-0.3699 \mathrm{Ok}$ \\
$-0,5559 \mathrm{Ok}$ \\
\end{tabular} & $\begin{array}{l}\text { Combinação quase permanente } \\
-0.5559 \mathrm{Ok} \\
-0.7348 \mathrm{OK} \\
-0.8692 \mathrm{Ok} \\
-0,9616 \mathrm{Ok} \\
-1,0143 \mathrm{Ok} \\
-1,0294 \mathrm{Ok} \\
-1,0143 \mathrm{Ok} \\
-0.9616 \mathrm{Ok} \\
-0.8692 \mathrm{Ok} \\
-0.7348 \mathrm{Ok} \\
-0,5559 \mathrm{Ok} \\
\end{array}$ & $\begin{array}{l}\text { Combinação frequente } \\
-0,5559 \mathrm{Ok} \\
-0,3256 \mathrm{Ok} \\
-0,1538 \mathrm{Ok} \\
-0,0375 \mathrm{Ok} \\
0,0257 \mathrm{Ok} \\
0,0376 \mathrm{Ok} \\
0,0257 \mathrm{Ok} \\
-0,0375 \mathrm{Ok} \\
-0,1538 \mathrm{Ok} \\
-0,3256 \mathrm{Ok} \\
-0,5559 \mathrm{Ok} \\
\end{array}$ & $\begin{array}{l}\text { Combinação frequente } \\
-0,5559 \mathrm{Ok} \\
-0,7749 \mathrm{Ok} \\
-0,9405 \mathrm{Ok} \\
-1,0552 \mathrm{Ok} \\
-1,1213 \mathrm{Ok} \\
-1,1409 \mathrm{Ok} \\
-1,1213 \mathrm{Ok} \\
-1,0552 \mathrm{Ok} \\
-0,9405 \mathrm{Ok} \\
-0,7749 \mathrm{Ok} \\
-0,5559 \mathrm{Ok} \\
\end{array}$ \\
\hline & & & \\
\hline
\end{tabular}

Figura 7: Verificação dos estados limites de serviço.

A Figura 8 mostra os valores das tensões normais no estado último e verifica se todos são menores que os valores máximos permitidos para tração e compressão. Além disso, também mostra a verificação da flecha. 


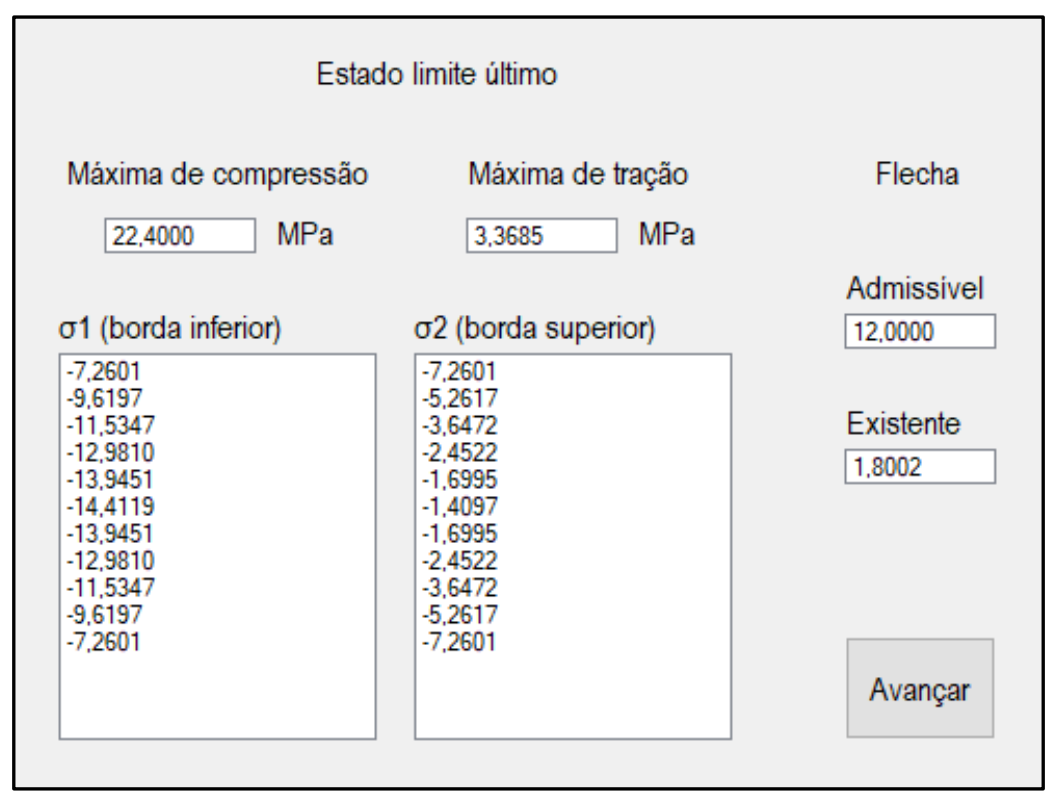

Figura 8: Verificação da flecha e do estado limite último.

A Figura 9 mostra o dimensionamento da armadura passiva e a verificação das bielas de compressão.

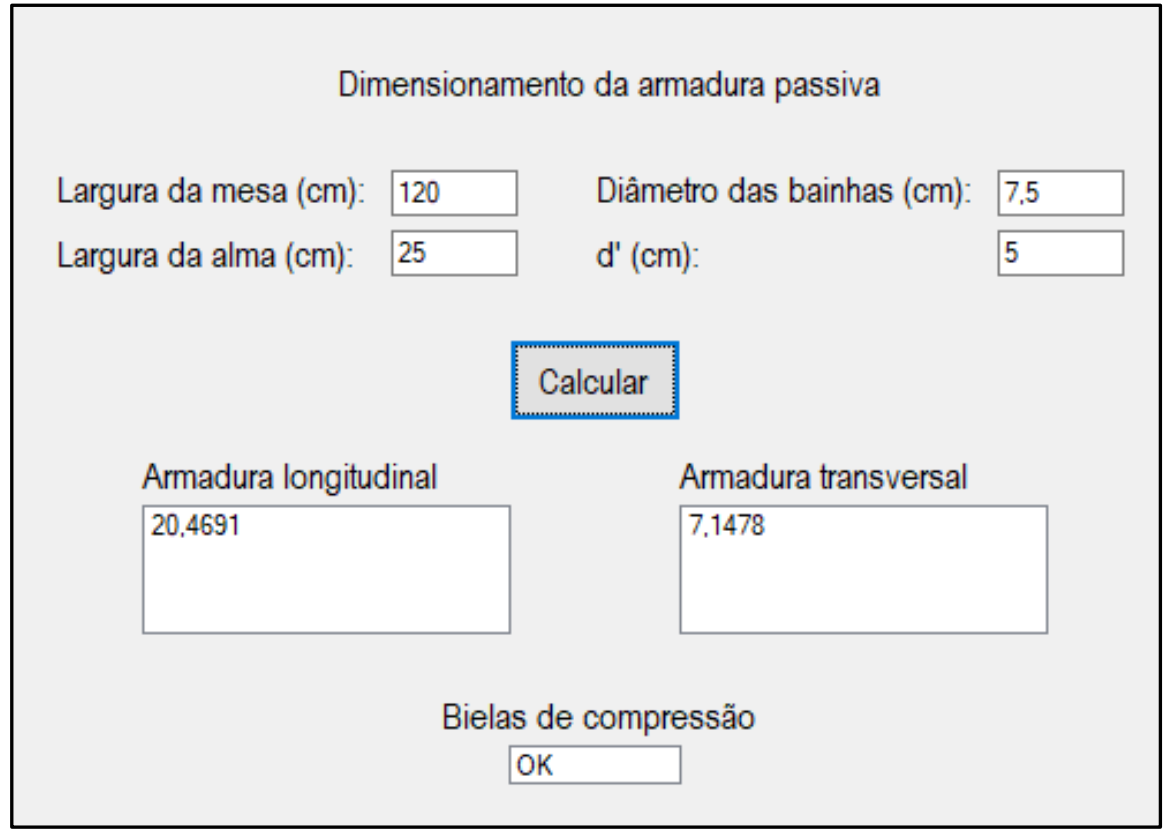

Figura 9: Dimensionamento da armadura passiva.

Os resultados obtidos através do programa foram validados a partir da comparação com o cálculo analítico, apresentando pequenas diferenças devido à arredondamentos neste, o que comprova que as rotinas implementadas até o momento estão funcionando corretamente. Por enquanto, a solução computacional realiza os cálculos referentes às propriedades geométricas da seção, força de protensão, área da armadura protendida, perdas de protensão, verificações de tensões normais em serviço, verificações de tensões normais em estado último no ato da protensão, verificação da flecha e área da armadura passiva. A ferramenta desenvolvida se mostrou eficaz e rápida para solução de problemas envolvendo vigas protendidas pós-tracionadas.

O programa possui uma interface que permite ao usuário a obtenção de resultados parciais que possibilita uma tomada de decisões mais adequada ao projeto. Por ser um programa aberto à comunidade, possibilita que se sejam realizadas novas implementações a fim de aumentar sua eficiência. 
As verificações mais importantes para vigas protendidas, tanto em relação aos estados limites últimos quanto em relação aos estados limites de serviço, são realizadas segundo a NBR - 6118: 2014 [5].

Essas verificações trazem valores adequados quando comparadas com os resultados apresentados por outros autores como Klein (2001) [10], Schwingel (1995) [7] e Moura (2004) [4]. Como o programa permite a leitura de resultados parciais, fica mais simples ao usuário tomar decisões mais precisas em relação ao projeto, segundo Araújo (2014) [15].

\section{CONCLUSÃO}

Algumas implementações serão incorporadas ao programa no futuro, como grau de protensão parcial, vigas pré-tracionadas, gráfico das perdas de protensão ao longo do comprimento da viga, outros traçados da armadura protendida e, também, detalhamento das peças em arquivos .dxf.

Pretende-se que sejam implementadas melhorias ao programa para que o mesmo possa ser utilizado tanto academicamente como em escritórios de projeto, a fim de tornar mais fácil a utilização desta técnica construtiva já largamente utilizada nas obras nacionais.

\section{REFERÊNCIAS BIBLIOGRÁFICAS}

1. Almeida SRM, Vaz LE, Éboli CR. Projeto ótimo de cabos de protensão em vigas. Anais da $29^{a}$ Jornada Sul Americana de Engenharia Estrutural; 2000; Punta del Este (UY).

2. Campos Filho A. Dimensionamento e verificação de seções poligonais de concreto armado submetidas à flexão composta oblíqua. Porto Alegre (RS): Universidade Federal do Rio Grande do Sul; 2014.

3. Franca GF. Sistema Computacional para Análise de Vigas em Concreto Armado e Protendido [dissertação]. Porto Alegre (RS): Universidade Federal do Rio Grande do Sul; 1999.

4. Moura M. Estudo sobre o traçado de cabos pós-tracionados em vigas isostáticas [dissertação]. Florianópolis (SC): Universidade Federal de Santa Catarina; 2004.

5. Associação Brasileira de Normas Técnicas. NBR 6118 - Projeto de estruturas de concreto Procedimento. Rio de Janeiro: ABNT; 2014.

6. Pfeil W. Concreto protendido - Introdução. Rio de Janeiro: Livros Técnicos e Científicos; 1988.

7. Schwingel R. Dimensionamento automático de vigas isostáticas, com protensão total ou parcial, por aderência inicial [dissertação]. Porto Alegre (RS): Universidade Federal do Rio Grande do Sul; 1995.

8. Silva IM, Takana ESA, Ishitani H. Sistematização do cálculo de perdas progressivas de protensão. Anais do $44^{\circ}$ Congresso Brasileiro do Instituto Brasileiro do Concreto; 17-22 Ago 2002; Belo Horizonte (MG).

9. Thiesen JR. Representação da Protensão por Carregamentos Equivalentes [dissertação]. Florianópolis (SC): Universidade Federal de Santa Catariana; 2001.

10. Klein R. Dimensionamento e detalhamento por computador de vigas simplesmente apoiadas de concreto protendido [dissertação]. Florianópolis (SC): Universidade Federal de Santa Catarina; 2001.

11.Zanette DS. Projeto de vigas de pequeno porte parcialmente protendidas com monocordoalhas engraxadas. Dissertação de Mestrado, Universidade Federal de Santa Catarina, 2006.

12. Momm JN. Dimensionamento Automático de Vigas Pré-Fabricadas em Concreto Protendido. Dissertação (Mestrado em Engenharia Civil) - Programa de Pós-Graduação em Engenharia Civil, Universidade Federal de Santa Catarina. 2004.

13. Koerich RB. Estudo de Estruturas Protendidas Hiperestáticas com a Representação da Protensão por Carregamentos Equivalentes. Dissertação. Programa de Pós-Graduação em Engenharia Civil, Universidade Federal de Santa Catarina, Florianópolis, 2004.

14. Collins MP; Mitchell D. Prestressed Concrete Basics. Canadian Prestressed Concrete Institute - CPCI. Ottawa, Ontario. 1987.

15. Araújo JM. Curso de concreto armado, Volume I, II, III e IV. Editora Dunas, 2014. 